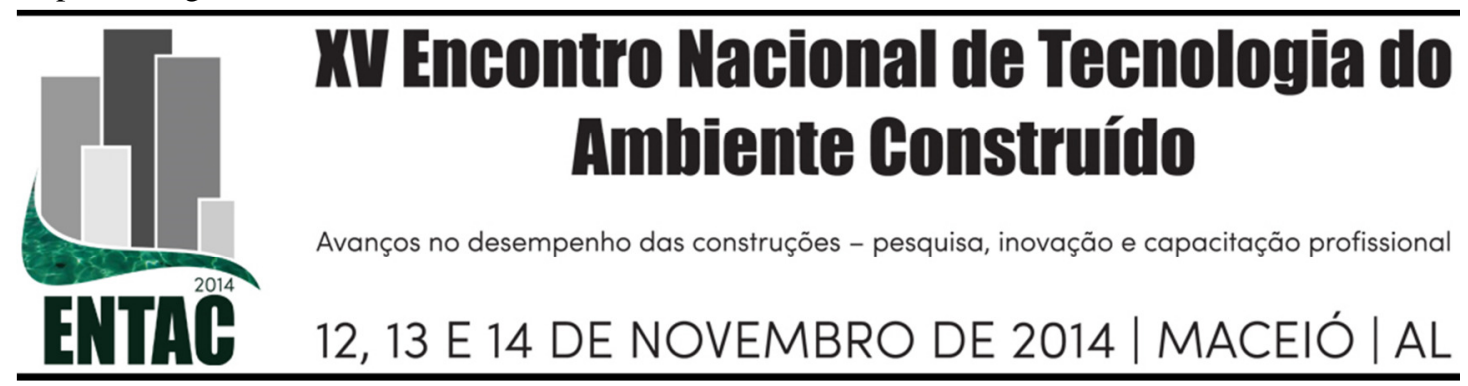

\title{
ANÁLISE DA SENSIBILIDADE DO MÉTODO PRESCRITIVO DO RTQ-C QUANTO À VARIAÇÃO DE DENSIDADE DE CARGA INTERNA
}

\author{
BRANDALISE, Mariane (1); AVILA, Vitória (2); OLIVEIRA, Larissa (3); \\ CUNHA, Eduardo (4) \\ (1)Arquiteta, mestranda do PROGRAU/UFPEL, marianebrandalise @ yahoo.com.br \\ (2) Acadêmica, curso de Arquitetura e Urbanismo/ UFPEL, vit_avila@yahoo.com.br \\ (3) Acadêmica, curso de Arquitetura e Urbanismo/ UFPEL, lari.sa.cris @ hotmail.com \\ (4) Prof. Dr. Arquiteto e Urbanista; Departamento de Tecnologia da Construção - Faculdade de \\ Arquitetura e Urbanismo/UFPel, eduardogralacunha@yahoo.com.br
}

\begin{abstract}
RESUMO
Alguns dos maiores avanços na área da eficiência energética de edificações no país nos últimos anos foram as publicações, do RTQ-C - Requisitos Técnicos da Qualidade para o Nível de Eficiência Energética de Edifícios Comerciais, de Serviços e Públicos, em 2009, e do RTQ-R - Regulamento Técnico da Qualidade para o Nível de Eficiência Energética de Edificações Residenciais, em 2010. Estes regulamentos definem metodologias de classificação das edificações em cinco níveis de eficiência. No caso do RTQ-C, a avaliação pode ser feita através de dois métodos: o método prescritivo, ou o método de simulação. A pesquisa tem como objetivo identificar a sensibilidade do Método Prescritivo do RTQ-C, quanto à variação de densidade de carga interna de equipamentos em edifícios de escritórios, na zona bioclimática 1 do zoneamento bioclimático brasileiro. O método do trabalho foi dividido em cinco etapas. $\mathrm{Na}$ primeira etapa foram definidos os modelos de análise; na segunda etapa foram caracterizadas as densidades de carga interna utilizadas na configuração dos modelos de análise, de acordo com a NBR 16401 (ABNT, 2008) e ASHRAE Fundamentals (2009). Na terceira etapa, foram determinadas as características da envoltória para os modelos de acordo com os pré-requisitos do RTQ-C. Na quarta etapa foi determinado o consumo energético dos modelos utilizando o software DesignBuilder, e na quinta e última etapa a análise dos resultados. Os resultados da pesquisa demonstram que a edificação com paredes e cobertura configuradas para atender aos pré-requisitos específicos para nível B, apresenta um consumo menor do que edificações que atendem aos pré-requisitos para nível A. O estudo também apresentou que edificações com elevada densidade de carga interna de equipamentos, fator de forma máximo e em altura, com paredes e coberturas com maior transmitância térmica, apresentam um consumo energético inferior a edificações com envoltória com maior resistência térmica. $\mathrm{O}$ aumento da densidade de carga interna provoca um acréscimo no calor interno, gerado pela grande quantidade de equipamentos. Em edificações com maior isolamento térmico (Nível A), o calor interno se mantem no ambiente, necessitando do sistema de ar condicionado para resfriamento.
\end{abstract}

Palavras-chave: Requisitos Técnico da Qualidade para o Nível de Eficiência Energética de Edifícios Comerciais, de Serviços e Públicos, Densidade de Carga Interna, Edifícios de Escritórios, Simulação Computacional, Eficiência Energética em Edificações.

\begin{abstract}
Some of the greatest advances in the energy efficiency of buildings in the country in the last years were the RTQ-C - Quality Technical Requirements for Energy Efficiency Level of Commercial, Service and Public Buildings, publications in 2009 and RTQ-R - Quality Technical Regulations for Energy Efficiency Level of Residential Buildings, in 2010.The regulations classify the buildings in five efficiency levels. In
\end{abstract}


RTQ-C, the evaluation can be done with two methods: a prescriptive method and a simulation one. The aim of the research is to identify the RTQ-C, sensibility towards the internal load diversity of equipments in the energy performance evaluation of office buildings envelopes situated in the bioclimatic zone one of Brazilian bioclimatic zoning. The research method was divided in five stages. At the first stage the analysis model was defined; at the second one, the internal load densities used in the analysis models settings, according to ASHRAE Fundamentals (2009), were characterized. At the third stage the envelope features for the models, according to efficiency levels A,B and C of RTQ-C were determined. In the fourth energy consumption of the models was determined using DesingBuilder software and, in the five and last stage the analysis of the results. The partial results of the research show that the building with walls and roof configured to answer the specific requirements for level B presents a lower consumption than buildings that answer to requirements for level A. The study also showed that buildings with high internal thermal load, maximum form factor and in height with wall and roofs with greater thermal transmittance show on energy consumption inferior to buildings with great thermal resistance.

Keywords: RTQ-C, internal load density, Office Buildings, computer simulation, Energy Efficiency in Buildings

\section{INTRODUÇÃO}

Os primeiros programas governamentais que buscavam eficiência energética em edificações surgiram na Europa e Estados Unidos, na década de 70. No Brasil, medidas neste sentido foram tomadas partir da década de 80. Mas o país somente buscou soluções mais eficazes vinculadas à eficientização energética de edificações no ano de 2001, após ocorrer o "apagão" energético. O primeiro passo foi à elaboração da Lei $\mathrm{N}^{\circ}$ 10.295 pelo Ministério de Minas e Energia, que entrou em vigor em 17 de outubro de 2001. Esta lei estabeleceu a criação de mecanismos que resultassem em edificações mais eficientes energeticamente (BRASIL, 2001).

Depois de alguns anos de discussão e de trabalhos, o Instituto Nacional de Metrologia, Qualidade e Tecnologia (INMETRO) publicou o RTQ-C - Requisitos Técnicos da Qualidade para o Nível de Eficiência Energética de Edifícios Comerciais, de Serviços e Públicos, em 2009, e o RTQ-R - Regulamento Técnico da Qualidade para o Nível de Eficiência Energética de Edificações Residenciais, em 2010. Os regulamentos classificam as edificações em cinco níveis de eficiência, a classificação varia sempre de $\mathrm{A}$ (mais eficiente) a $\mathrm{E}$ (menos eficiente).

No caso do RTQ-C, a avaliação poderá ser feita através de dois métodos: o método prescritivo, que é um método simplificado, ou o método de simulação. O Método prescritivo foi desenvolvido através da utilização do método estatístico de regressão linear múltipla, baseando-se em resultados de consumo final de diferentes edificações comerciais, gerados através do uso da simulação computacional. Pelo Método Prescritivo, utilizam-se duas equações por Zona Bioclimática, uma para edificações com área de projeção menor que $500 \mathrm{~m}^{2}$, e com Fator de Forma Máximo, e outra para edificação maior que $500 \mathrm{~m}^{2}$, e com Fator de Forma Mínimo. O Fator Forma é determinado pela razão entre a área de envoltória e o volume total da edificação. Os métodos simplificados provêm uma ferramenta rápida para a avaliação do desempenho da edificação, porém podem envolver uma incerteza considerável em seus resultados, levando a comprometer o processo de certificação dos edifícios (MELO, 2012).

No caso do desempenho energético das edificações de escritórios uma série de aspectos devem ser considerados. Alguns deles, como a envoltória da edificação, o sistema de iluminação artificial como também o sistema de condicionamento de ar, são considerados no RTQ-C. Entretanto a densidade de carga interna gerada por equipamentos é um dos fatores que interfere no desempenho energético das edificações não é considerado no método prescritivo do RTQ-C. No caso de edifícios de escritórios, 
onde se observa uma variação muito grande quanto à ocupação, a DCI, dependendo do uso dos ambientes, pode interferir signficamente.

De acordo com Westphal (2007), para um modelo de edifício de escritório na cidade de Curitiba, com a densidade de carga interna de $20 \mathrm{~W} / \mathrm{m}^{2}$, baixa absortância superficial externa, e padrão de uso de $8 \mathrm{~h} /$ dia, o aumento da transmitância térmica, aumenta o consumo de energia elétrica em climatização. No entanto, na mesma edificação com uma densidade de carga interna de $50 \mathrm{~W} / \mathrm{m}^{2}$, percebe-se uma redução no consumo de energia elétrica em climatização quando a envoltória apresenta uma maior transmitância térmica.

No âmbito que está inserida está pesquisa, pretende-se identificar a sensibilidade do método prescritivo do RTQ-C, quanto à variação de densidade de carga interna (DCI) de equipamentos, na avaliação do desempenho energético da envoltória de edifícios de escritórios localizados Zona Bioclimática 1 do Zoneamento Bioclimático Brasileiro.

\section{MÉTODO}

\subsection{Definição dos modelos de análise}

Para este estudo foram definidos três modelos de análise. O primeiro modelo analisado foi definido com base no estudo desenvolvido por Carlo (2008). Onde, através de um levantamento fotográfico, pode observar volumetrias típicas, mais frequentes na paisagem urbana, para algumas atividades comerciais. A partir do levantamento, foi possível gerar um modelo representativo para cada atividade. $O$ modelo 1 foi identificado pela autora com o nome de grandes escritórios e caracterizado como edificação vertical, área de pavimento tipo menor que $500 \mathrm{~m}^{2}$, possuindo 5 pavimentos, forma retangular, com dimensão de $27 \mathrm{~m}$ de comprimento, $7,8 \mathrm{~m}$ de largura e $15 \mathrm{~m}$ de altura. A Figura 1 ilustra o modelo 1.

\section{Figura 1 - Modelo 1}

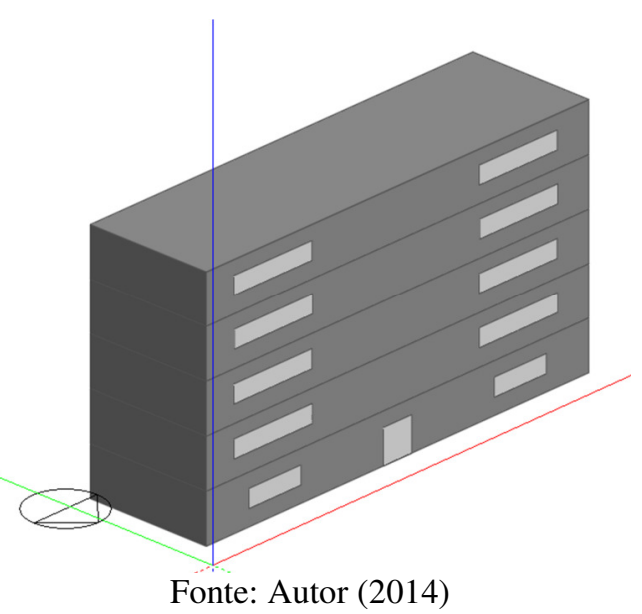

Os outros dois modelos estudados foram determinados com base nas equações do RTQC, que limita um Fator de Forma mínimo e um Fator de Forma máximo. O Fator Forma é determinado pela razão entre a área de envoltória e o volume total da edificação. Desta forma foi definido um segundo modelo com área de projeção menor que $500 \mathrm{~m}^{2}$, Fator 
de Forma 0,60 (Fator de Forma máximo para zona bioclimática 1), com 10 pavimentos e dimensões de 10 metros de comprimento, 6 metros de largura e 30 metros de altura. $\mathrm{E}$ um terceiro modelo também com área de projeção menor que $500 \mathrm{~m}^{2}$ e Fator de Forma 0,60 (Fator de Forma máximo), porém com um só pavimento e dimensões de 27 metros de comprimento, 7,5 metros de largura e 4 metros de altura. Estes dois modelos foram escolhidos com intuito de avaliar as diferenças entre uma área maior de envoltória na cobertura e uma área maior de paredes. Na Figura 2 e na Figura 3, a seguir podem ser observados os modelos 2 e 3 , respectivamente.

Figura 2 - Modelo 2

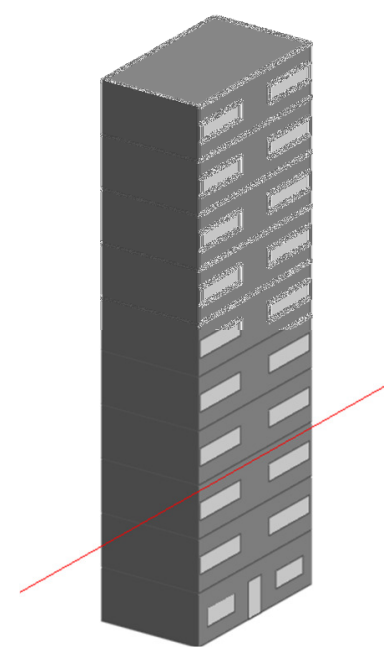

Fonte: Autor (2014)
Figura 3 - Modelo 3

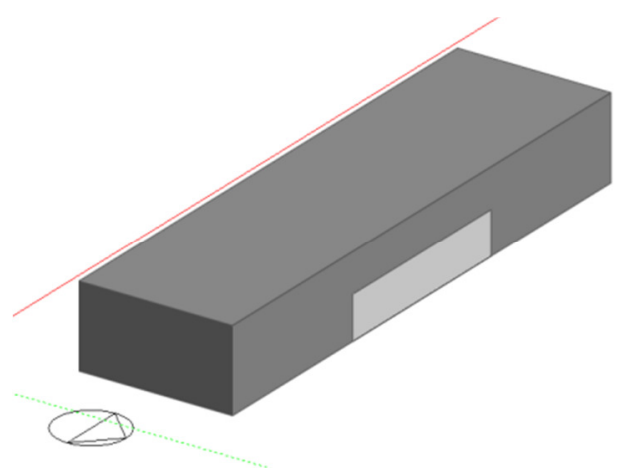

Fonte: Autor (2014)

\subsection{Caracterização da Densidade de Carga Interna a ser utilizada na configuração dos modelos de análise.}

A densidade de carga interna é definida pela soma das três principais fontes de calor internas: iluminação, equipamentos elétricos e pessoas $\mathrm{O}$ sistema de iluminação dos modelos, foram configurados com a densidade de potência de iluminação $10 \mathrm{~W} / \mathrm{m}^{2}$. O RTQ-C classifica nível A o sistema de iluminação para edifícios de escritório com potência limite de $9,7 \mathrm{~W} / \mathrm{m}^{2}$. Porém, o software DesignBuilder possibilita somente a utilização de números inteiro, por esse motivo o valor foi arredondado para $10 \mathrm{~W} / \mathrm{m}^{2}$. O sistema de condicionamento de ar dos modelos foi considerado do tipo split com coeficiente de performance (COP) de 3,21, considerado nível A, pelo RTQ-C.

A norma ASHRAE Fundamentals (2009), classifica os escritórios em quatro tipos de densidade de carga interna de equipamentos. Neste trabalho foram avaliados somente dois tipos de densidade, a média e a alta. A Tabela 1 caracteriza os valores adotados de acordo com a Norma ASHRAE Fundamentals (2009) para a densidade de carga interna de equipamentos, NBR 16.401- Parte 3 para densidade de pessoas e RTQ-C para a potência de iluminação. 
Tabela 1 - Caracterização das DCI utilizadas nos modelos de análise

\begin{tabular}{|c|c|c|c|}
\hline Tipo de Escritório & $\begin{array}{c}\text { DCI } \\
\text { Equipamentos }\end{array}$ & $\begin{array}{c}\text { Densidade de } \\
\text { Pessoas }\end{array}$ & $\begin{array}{c}\text { Potência de } \\
\text { iluminação }\end{array}$ \\
\hline Média densidade & $11 \mathrm{~W} / \mathrm{m}^{2}$ & $0,14\left(\right.$ pessoas $\left./ \mathrm{m}^{2}\right)$ & $10\left(\mathrm{~W} / \mathrm{m}^{2}\right)$ \\
\hline Alta densidade & $21 \mathrm{~W} / \mathrm{m}^{2}$ & $0,20\left(\right.$ pessoas $\left./ \mathrm{m}^{2}\right)$ & $10\left(\mathrm{~W} / \mathrm{m}^{2}\right)$ \\
\hline
\end{tabular}

Fonte: Autor (2014)

\subsection{Determinação das características da envoltória da edificação}

O RTQ-C determina pré-requisitos específicos da envoltória para cada Zona Bioclimática, que devem ser atendidos de acordo com o nível de eficiência que se pretende alcançar. Os modelos foram configurados de acordo com os pré-requisitos para Zona Bioclimática 1. Na Zona Bioclimática 1, a absortância não é considerada um prérequisito da envoltória. Já nas Zonas Bioclimáticas 2 a 8 , para obter-se nível A deve-se utilizar materiais de revestimento externo com absortância solar menor que 0,50 $(\alpha<$ $0,50)$ em paredes e coberturas. Para o nível B o pré-requisito absortância solar de 0,50 é definido apenas para a cobertura. Para nível C e D não há esse pré-requisito. Optou-se em utilizar a absortância de acordo com os pré-requisitos das Zonas Bioclimáticas 2 a 8 , já que a Zona Bioclimática 1 é livre, conforme pode ser observado na Tabela 2, podendo, portanto, observar também o impacto da cor dos fechamentos nas análises propostas.

Tabela 2 - Características da envoltória dos modelos de análise

\begin{tabular}{|c|c|c|c|}
\hline Pré -Requisitos Envoltória & Nível A & Nível B & Nível C \\
\hline $\begin{array}{c}\text { Transmitância Térmica } \\
\text { Cobertura }\end{array}$ & $\begin{array}{c}0,5 \\
\left(\mathrm{~W} / \mathrm{m}^{2} \cdot \mathrm{K}\right)\end{array}$ & $\begin{array}{c}1,0 \\
\left(\mathrm{~W} / \mathrm{m}^{2} \cdot \mathrm{K}\right)\end{array}$ & $\begin{array}{c}2,0 \\
\left(\mathrm{~W} / \mathrm{m}^{2} \cdot \mathrm{K}\right)\end{array}$ \\
\hline $\begin{array}{c}\text { Transmitância Térmica } \\
\text { Parede }\end{array}$ & $\begin{array}{c}1,0 \\
\left(\mathrm{~W} / \mathrm{m}^{2} \cdot \mathrm{K}\right)\end{array}$ & $\begin{array}{c}2,0 \\
\left(\mathrm{~W} / \mathrm{m}^{2} \cdot \mathrm{K}\right)\end{array}$ & $\begin{array}{c}3,7 \\
\left(\mathrm{~W} / \mathrm{m}^{2} \cdot \mathrm{K}\right)\end{array}$ \\
\hline Absortância Cobertura & 0,20 & 0,20 & 0,20 \\
\hline Absortância Parede & 0,20 & 0,20 & 0,70 \\
\hline
\end{tabular}

Fonte: Autor (2014)

Pelo método prescritivo, o Percentual de Abertura na Fachada total (PAFt) é considerado uma das variáveis no cálculo do indicador de consumo da envoltória. Não sendo considerado, portanto, um pré-requisito da envoltória. Como este trabalho analisa os diferentes níveis de eficiência energética do RTQ-C (A,B, C e D), utilizou-se o método do calculo dos PAFTs dos edifícios de referência, o qual é utilizado no método de simulação, para encontrar o PAFT limite para cada nível de eficiência em cada modelo. Sendo assim, para o cálculo aplicou-se a equação do indicador de consumo da envoltória (ICenv) isolando a variável PAFT, como pode ser observado na Equação 1 a seguir.

Equação 1:

$\mathrm{PAFT}<\mathrm{R}>=\mathrm{ICR}+43,0 . \mathrm{FA}+316,62 . \mathrm{FF}-7,29 . \mathrm{FS}-132,5 \mathrm{FA} / \mathrm{FF}+77 \mathrm{FA} . \mathrm{FF}-182,66$

16,38

onde, $\quad$ ICR = Indicador de consumo do modelo de referência (adimensional) 
$\mathrm{FA}=$ Fator altura (Apco/Atot)

$\mathrm{FF}=$ Fator de forma (Aenv/Vtot) equação)

PAFT $=$ Percentual de abertura na fachada total (adimensional, para uso na

$\mathrm{FS}=$ Fator solar

Apcob $=$ Área de projeção da cobertura em $\left(\mathrm{m}^{2}\right)$

Aenv $=$ Área da envoltória $\left(\mathrm{m}^{2}\right)$

Atot $=$ Area total de piso $\left(\mathrm{m}^{2}\right)$

Vtot $=$ Volume total de piso $\left(\mathrm{m}^{3}\right)$

Sendo assim os modelos foram configurados com um PAFT inicial de 0,05, e sofreram variações de acréscimo de 0,05 , até o PAFT limite para cada nível de eficiência.

\subsection{Determinação do consumo dos modelos com características para nível A, B e C.}

Para se obter o consumo dos edifícios com características de acordo com diferentes níveis de eficiência energética do RTQ-C foram feitas simulações no software DesignBuilder versão 3.0.0.15. Inicialmente foi configurado o arquivo climático PR_Curitiba.epw para a cidade de Curitiba, Zona Bioclimática 1. Após a modelagem das edificações no software, foram informados os parâmetros utilizados nas simulações, conforme pode ser observado na Tabela 4.

Tabela 4 -Parâmetros utilizados nos modelos de análise

\begin{tabular}{|c|c|}
\hline Parâmetros & Valores Adotados \\
\hline $\begin{array}{l}\text { Padrão de uso (h/dia) (ocupação, equipamentos e } \\
\text { Iluminação). } \\
\text { Sábados e domingos não foram considerados como } \\
\text { períodos de ocupação }\end{array}$ & $\begin{array}{c}08: 00 \text { até às } 12: 00-100 \% \\
12: 00 \text { até às } 14: 00-10 \% \\
14: 00 \text { até às } 18: 00-100 \%\end{array}$ \\
\hline $\begin{array}{l}\text { Coeficiente de Performance do sistema de } \\
\text { condicionamento de ar (W/W) }\end{array}$ & $\begin{array}{l}3,21 \mathrm{~W} / \mathrm{W} \text { para aquecimento e } \\
\text { resfriamento }\end{array}$ \\
\hline Setpoint de aquecimento $\left({ }^{\circ} \mathrm{C}\right)$ & $18^{\circ} \mathrm{C}$ \\
\hline Setpoint de resfriamento $\left({ }^{\circ} \mathrm{C}\right)$ & $24^{\circ} \mathrm{C}$ \\
\hline Orientação da maior fachada & Norte/ Sul \\
\hline
\end{tabular}

Fonte: Autor (2014)

\subsection{Comparação do consumo dos edifícios com envoltória nível $A, B$ e $C$ com diferentes densidades de carga interna de equipamento}

A partir das simulações foi obtido o consumo energético anual de cada modelo. Com esses dados foi possível avaliar e comparar o desempenho energético para as diferentes configurações da envoltória de acordo com o RTQ-C. Como resultados, serão apresentadas análises comparativas das variadas simulações em forma de gráficos de consumo anual com relação aos níveis de eficiência do RTQ-C. 


\section{ANALISE DOS RESULTADOS}

Os gráficos abaixo apresentam os resultados do consumo energético para densidade média e alta por ano de cada modelo. A comparação dos resultados foi realizada até o PAFT 0,15, pois este é o PAFT limite para o Nível A, sendo estes PAFTs $(0,05 ; 0,10$ e $0.15)$ presentes nos diferentes níveis de eficiência energética analisados.

\section{Gráfico 1-Resultado do consumo anual do modelo 1 DCI de equipamento $11 \mathrm{~W} / \mathrm{m}^{2}$}

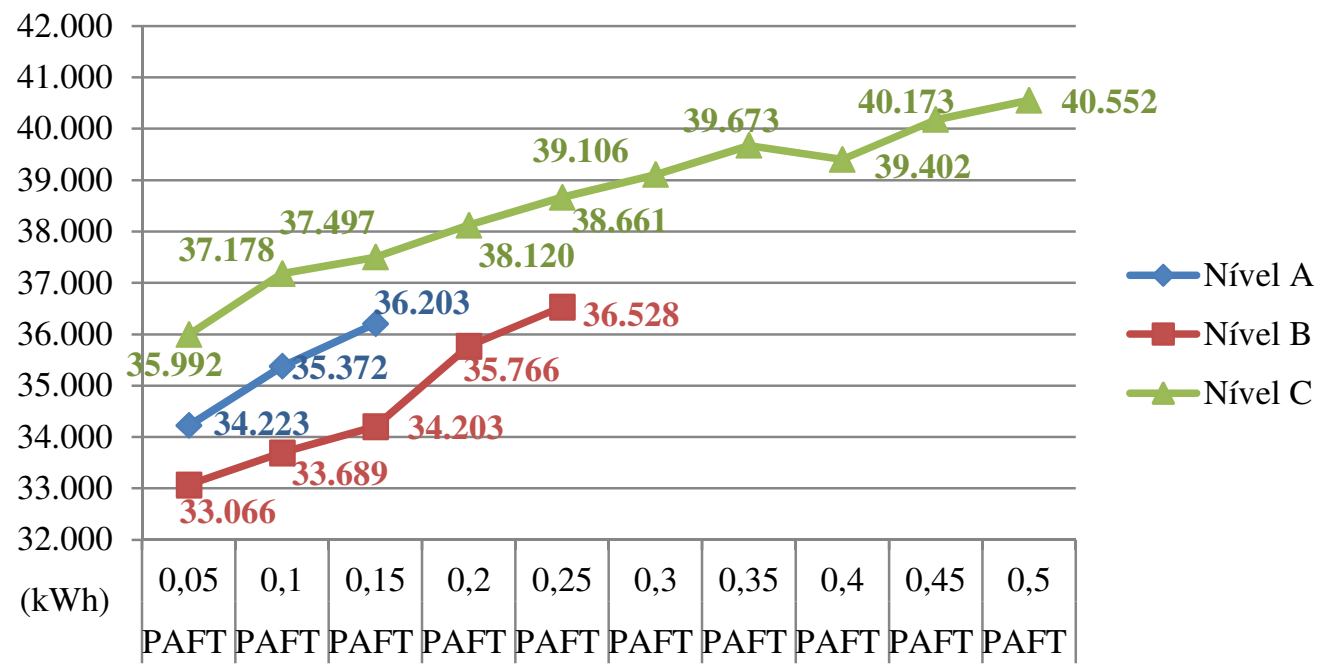

Fonte: Autor (2014)

\section{Gráfico 2-Resultado consumo anual modelo 1 DCI de equipamento $21 \mathrm{~W} / \mathrm{m}^{2}$}

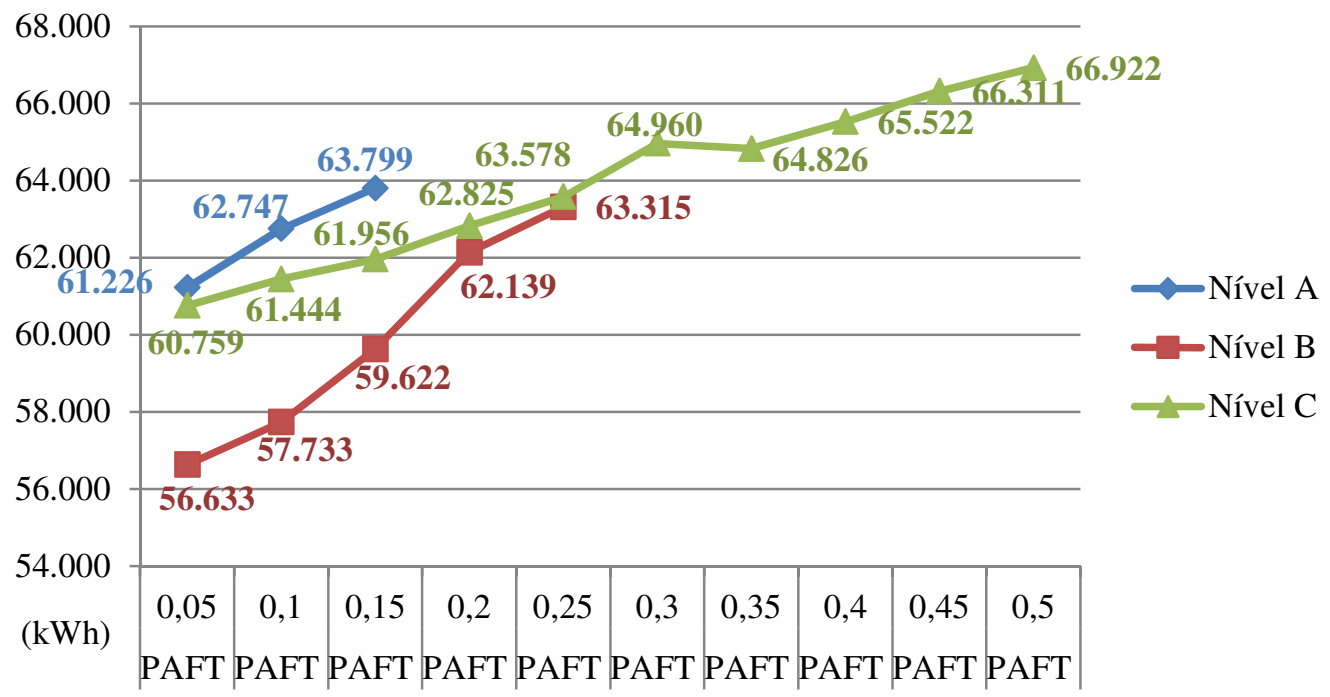

Fonte: Autor (2014)

Para o primeiro modelo analisado, com a densidade de equipamentos $11 \mathrm{~W} / \mathrm{m}^{2}$, os exemplares atendendo aos pré-requisitos para nível $\mathrm{B}$, apresentaram um consumo energético inferior aos exemplares atendendo aos pré-requisitos para nível A, conforme o gráfico 1. O exemplar com PAFT 0,15, foi o que apresentou maior diferença de consumo entre níveis A e B, sendo esta diferença de 6,30\% a mais para o nível A.

Já com a densidade de equipamentos de $21 \mathrm{~W} / \mathrm{m}^{2}$ (gráfico 2), o exemplar que apresentou maior diferença de consumo entre os níveis A e B, foi o modelo atendendo aos prérequisitos para nível A com PAFT 0,10. O qual demonstrou um consumo de 7,99\% a 
mais que o exemplar atendendo aos pré-requisitos para nível B com PAFT 0,10. Analisando a diferença de consumo entre as amostras, atendendo aos pré-requisitos para nível A e nível $\mathrm{C}$, podemos observar que a amostra atendendo aos pré-requisitos para nível A com PAFT 0,10 apresentou um consumo de 6,38\% maior do que o modelo atendendo aos pré-requisitos para nível C com PAFT 0,10.

\section{Gráfico 3-Resultado consumo anual modelo 2 DCI de equipamento $11 \mathrm{~W} / \mathrm{m}^{2}$}

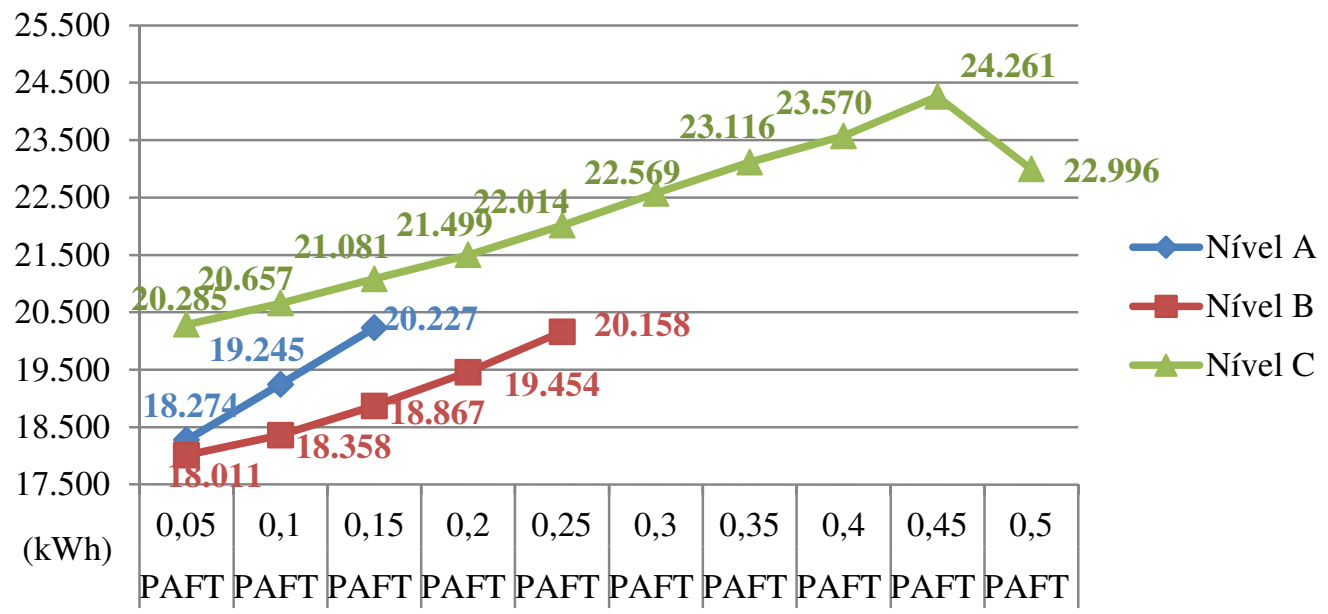

Fonte: Autor (2014)

\section{Gráfico 4-Resultado consumo anual modelo 2 DCI de equipamento $21 \mathrm{~W} / \mathrm{m}^{2}$}

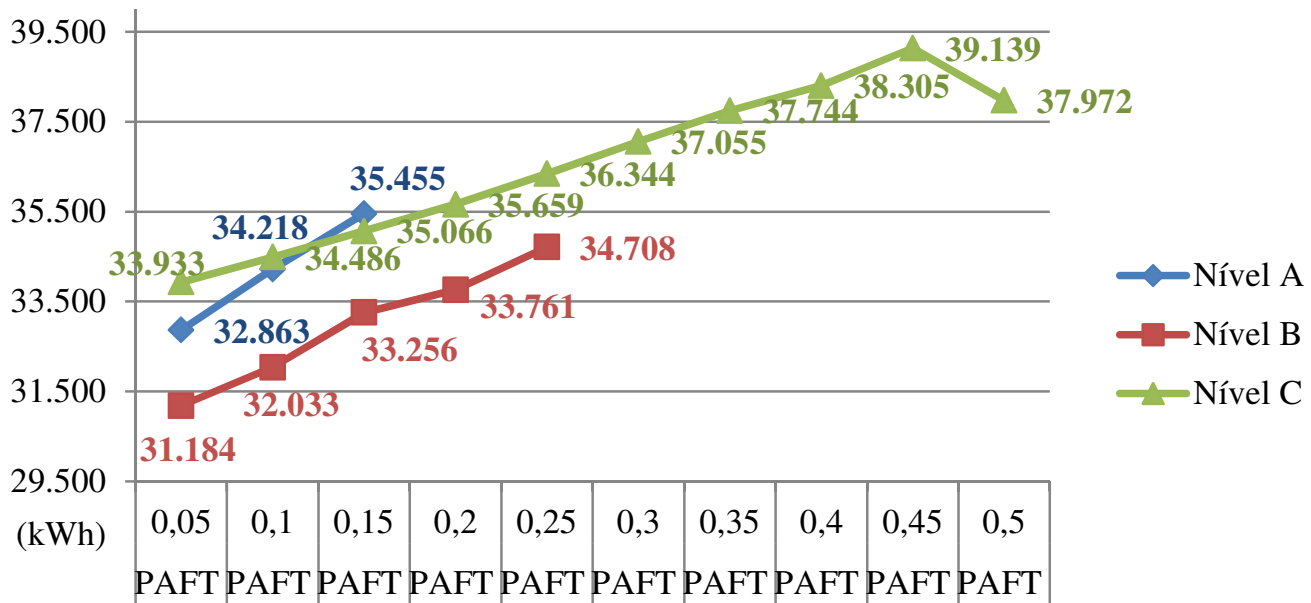

Fonte: Autor (2014)

Para os resultados do segundo modelo analisado, pode ser observado, que com densidade de equipamentos de $11 \mathrm{~W} / \mathrm{m}^{2}$ (Gráfico 3), o exemplar de PAFT 0,15 , que atende aos pré-requisitos para nível $\mathrm{A}$, apresentou um consumo maior em $6,72 \%$ do que o exemplar atendendo aos pré-requisitos para nível B. Sendo essa a maior diferença de consumo entre os modelos de nível A e nível B. Nos exemplares com densidade de equipamentos de $21 \mathrm{~W} / \mathrm{m}^{2}$ (Gráfico 4), percebe-se a diferença de consumo, entre os modelo que atendem aos pré-requisitos para nível A e B no exemplar de PAFT 0,10, em torno de 6,38\%. Considerando os resultados entre os modelos atendendo aos prérequisitos para nível $\mathrm{A}$ e atendendo aos pré-requisitos para nível $\mathrm{C}$, percebe-se que apenas no modelo de PAFT 0,15, o modelo com envoltória para nível C apresenta um 
consumo energético inferior em relação ao modelo com envoltória para nível A, sendo esta diferença pequena, apenas $1,09 \%$.

\section{Gráfico 5 -Resultado consumo anual modelo 3 DCI de equipamento $11 \mathrm{~W} / \mathrm{m}^{2}$}

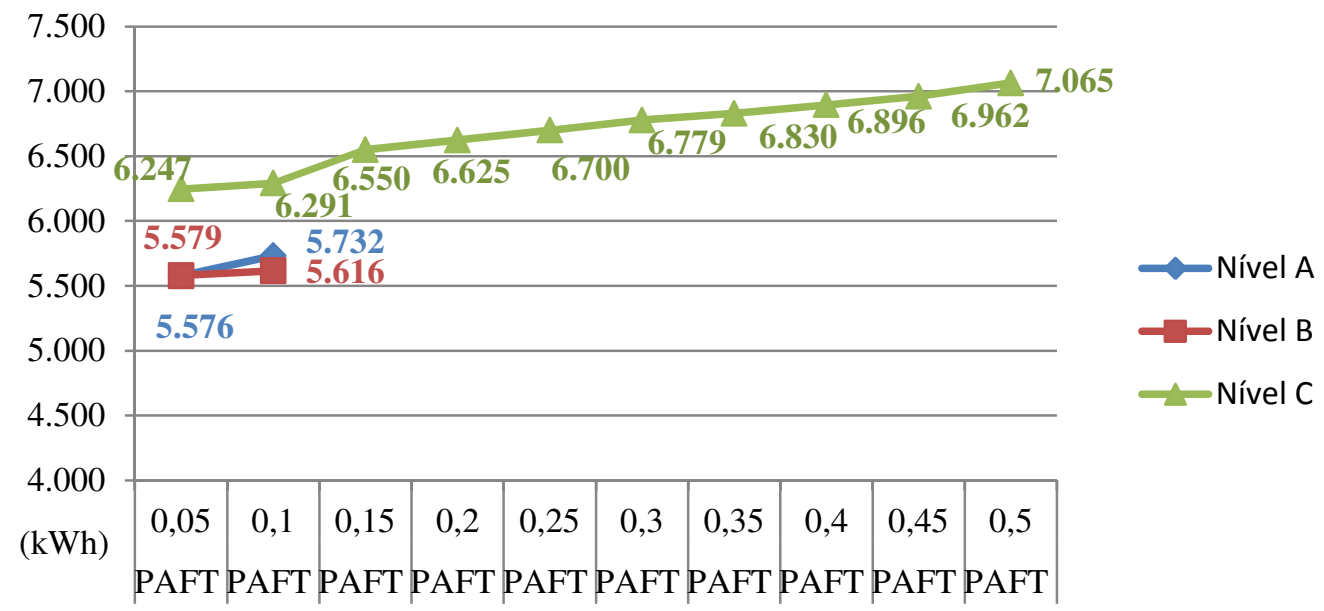

Fonte: Autor (2014)

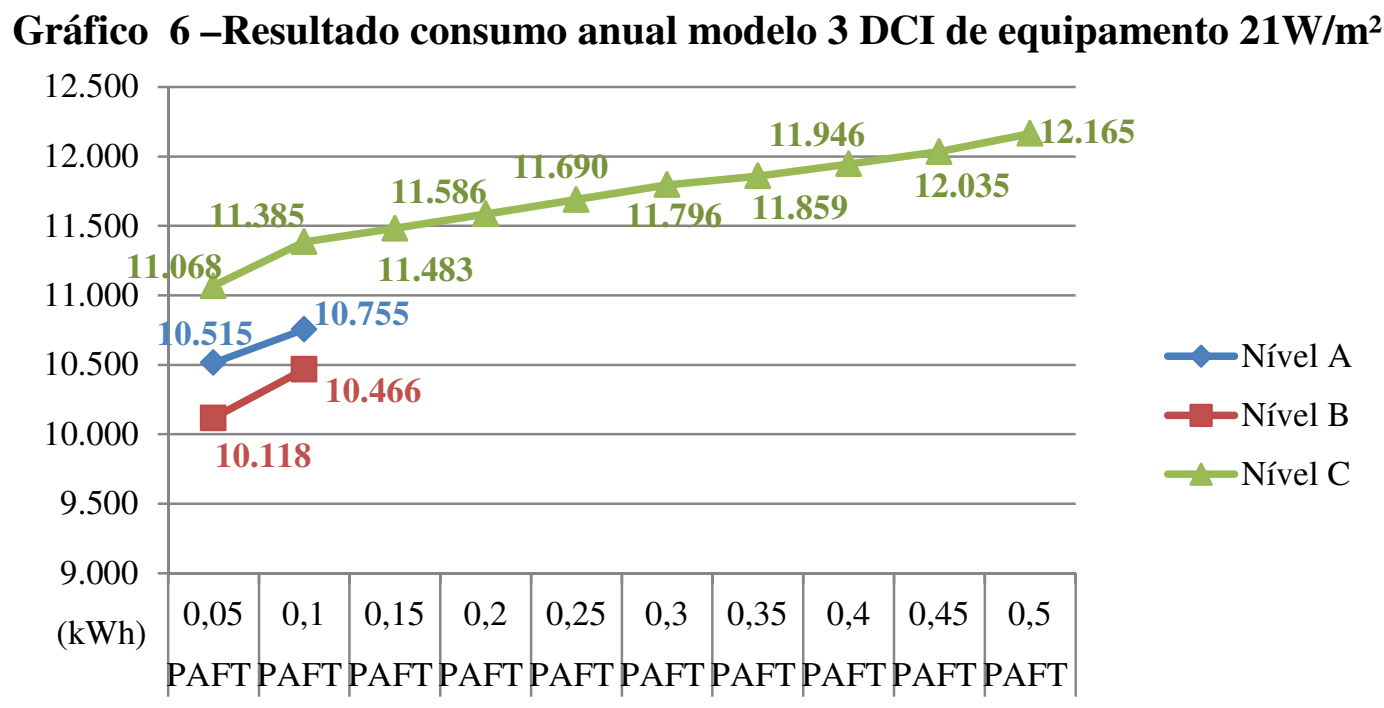

Fonte: Autor (2014)

No terceiro modelo investigado, com densidade de equipamentos de $11 \mathrm{~W} / \mathrm{m}^{2}$ (Gráfico 5), apenas um modelo atendendo aos pré-requisitos para nível A, apresentou consumo superior aos modelos atendendo aos pré-requisitos para nível B. Esse comportamento pode ser observado no modelo de PAFT 0,10, o qual apresentou um consumo energético de $1,84 \%$, maior do que o modelo atendendo aos pré-requisitos para nível $\mathrm{B}$. Já nos exemplares configurados com a densidade de equipamentos de $21 \mathrm{~W} / \mathrm{m}^{2}$ (Gráfico 6), a maior diferença de consumo, entre os modelos atendendo aos pré-requisitos para nível A e atendendo aos pré-requisitos para nível $\mathrm{B}$, pode ser percebida no modelo de PAFT 0,05. O modelo atendendo aos pré-requisitos para nível A apresentou um consumo de $3,77 \%$ superior ao modelo configurado de acordo com os pré-requisitos para o nível $\mathrm{B}$. Os modelos atendendo aos pré-requisitos para nível $\mathrm{C}$ apresentaram maior consumo entre os três níveis, nas duas densidades de equipamentos analisadas. 


\section{CONCLUSÕES OU CONSIDERAÇÕES FINAIS}

Os novos regulamentos de eficiência energética consideram a variação da DCI no caso da iluminação artificial apenas. Não existe qualquer observação do RTQ-C, para o caso de edifícios de escritórios, quanto às possibilidades de ocupação e geração de calor.

O trabalho demonstrou que edifícios com elevada DCI e um maior isolamento da envoltória podem obter maior consumo energético. Observou-se que para a Zona Bioclimática 1 a edificação com paredes e cobertura configuradas para atender aos prérequisitos específicos para nível $\mathrm{B}$ (transmitância térmica de paredes e cobertura) apresenta um consumo inferior a envoltória que atende ao nível $\mathrm{A}$.

Em relação à diferença de altura das edificações, pode-se perceber, que edificações com elevada DCI, fator de forma máximo, em altura, e com envoltória atendendo aos prérequisitos específicos para nível $\mathrm{C}$, podem apresentam um consumo energético inferior à edificações com envoltória com maior resistência térmica (pré-requisitos específicos para nível A) como observado no modelo 1 e 2.

As análises realizadas, considerando-se a Zona Bioclimática 1, evidenciam a importância da avaliação da variação DCI de equipamentos em conjunto com outras variáveis, como fator de forma mínimo e máximo. O estudo demonstrou a necessidade de revisão das transmitâncias térmicas de paredes e coberturas definidas como prérequisitos específicos no método prescritivo do RTQ-C em decorrência das possibilidades de edifícios com elevadas DCI.

\section{REFERÊNCIAS}

ASHRAE - AMERICAN SOCIETY OF HEATING, REFRIGERATING AND AIRCONDITIONING ENGINEERS. 2009 ASHRAE Handbook -Fundamentals. SI Edition p. 9.19 Atlanta, 2009.

BRASIL. Lei n. 10295, de 17 de outubro de 2001. Dispõe sobre a Política Nacional de Conservação e Uso Racional de Energia. Lex: Diário Oficial da União, Brasília, $2001 \mathrm{a}$.

Decreto n. 4.059, de 19 de dezembro de 2001. Regulamenta a Lei no 10.295, de 17 de outubro de 2001, que dispõe sobre a Política Nacional de Conservação e Uso Racional de Energia, e dá outras providências. Lex: Diário Oficial da União, Brasília, 2001b.

CARLO, J. Desenvolvimento de Metodologia de Avaliação da Eficiência Energética do Envoltório de Edificações não Residenciais. 2008. Tese de doutorado, Programa de PósGraduação em Engenharia Civil - Universidade Federal de Santa Catarina, Florianópolis.

MELO, Ana Paula. Desenvolvimento de um método para estimar o consumo de energia de edificações comerciais através da aplicação de redes neurais. 2012. Tese (Doutorado em Engenharia Civil)- Programa de Pós-Graduação em Engenharia Civil, Universidade Federal de Santa Catarina, Florianópolis.

INMETRO - INSTITUTO NACIONAL DE METROLOGIA, NORMALIZAÇÃO E QUALIDADE INDUSTRIAL. Requisitos Técnicos da Qualidade para o Nível de Eficiência Energética de Edifícios Comerciais, de Serviços e Públicos. Eletrobrás, 2013.

WESTPHAL, F. Análise de Incertezas e de Sensibilidade Aplicadas à simulação de Desempenho Energético de Edificações Comerciais. 2007. Tese de doutorado, Programa de Pós-Graduação em Engenharia Civil - Universidade Federal de Santa Catarina, Florianópolis. 